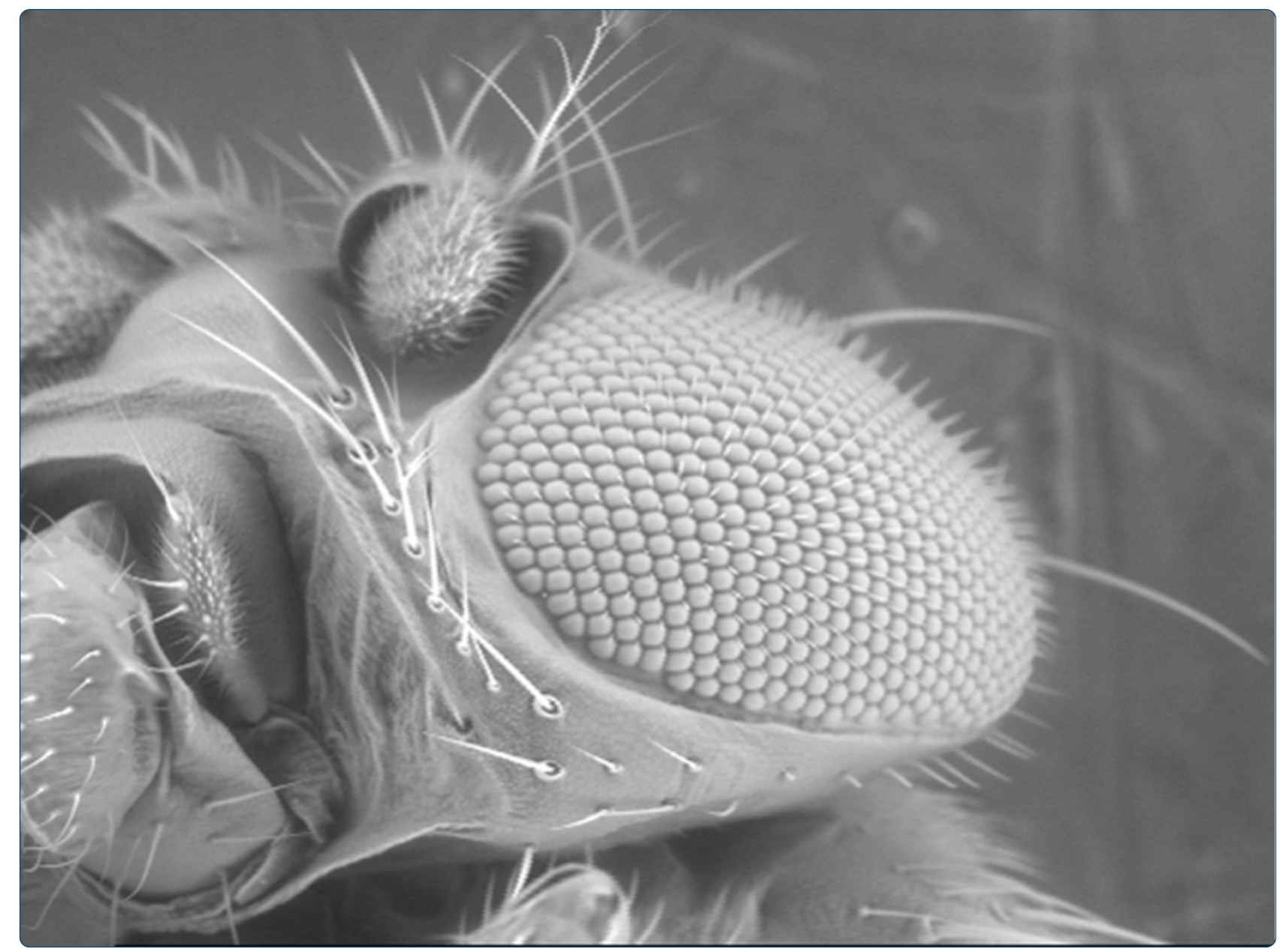

\title{
A role for the membrane protein M6 in the Drosophila visual system
}

Zappia et al.

C Biomed Central 


\title{
A role for the membrane protein M6 in the Drosophila visual system
}

\author{
María Paula Zappia ${ }^{1} 2^{*}$, Guillermo Bernabo ${ }^{2}$, Silvia C Billi ${ }^{1}$, Alberto C Frasch ${ }^{1}$, María Fernanda Ceriani ${ }^{2^{*}}$ \\ and Marcela Adriana Brocco ${ }^{1}$
}

\begin{abstract}
Background: Members of the proteolipid protein family, including the four-transmembrane glycoprotein M6a, are involved in neuronal plasticity in mammals. Results from our group previously demonstrated that M6, the only proteolipid protein expressed in Drosophila, localizes to the cell membrane in follicle cells. M6 loss triggers female sterility, which suggests a role for M6 in follicular cell remodeling. These results were the basis of the present study, which focused on the function and requirements of M6 in the fly nervous system.

Results: The present study identified two novel, tissue-regulated M6 isoforms with variable N- and C- termini, and showed that M6 is the functional fly ortholog of Gpm6a. In the adult brain, the protein was localized to several neuropils, such as the optic lobe, the central complex, and the mushroom bodies. Interestingly, although reduced M6 levels triggered a mild rough-eye phenotype, hypomorphic M6 mutants exhibited a defective response to light.

Conclusions: Based on its ability to induce filopodium formation we propose that M6 is key in cell remodeling processes underlying visual system function. These results bring further insight into the role of M6/M6a in biological processes involving neuronal plasticity and behavior in flies and mammals.
\end{abstract}

Keywords: Myelin PLP family, Gpm6a, Eye development, Phototactic behavior, Lifespan, Protrusion/filopodium formation, Cell remodeling

\section{Background}

Neural plasticity is the mechanism by which information is stored and maintained within individual synapses, neurons, and neuronal circuits to guide organism behavior. Neurite growth and remodeling represents a fundamental process during nervous system development, plasticity, and behavior. Although neuronal plasticity allows the organism to adapt to a constantly evolving environment, little is known about the molecular components and pathways that support it. Several myelin proteolipid protein (PLP) family members, such as M6a, M6b, and DM20 [1], have been shown to be involved in

\footnotetext{
* Correspondence: mpzappia@iib.unsam.edu.ar; fceriani@leloir.org.ar "Instituto de Investigaciones Biotecnológicas "Dr. Rodolfo Ugalde", Instituto Tecnológico de Chascomús, Consejo Nacional de Investigaciones Científicas y Técnicas, Universidad Nacional de San Martín, IIB, INTECH, CONICET-UNSAM , 25 de Mayo y Francia, Edificio IIB, B1650HMP, San Martín, Provincia de Buenos Aires, Argentina

${ }^{2}$ Laboratorio de Genética del Comportamiento, Fundación Instituto Leloir and Instituto de Investigaciones Bioquímicas, Buenos Aires (IIB-BA, CONICET), Fundación Instituto Leloir, Av. Patricias Argentinas 435, 1405BWE, CABA, Buenos Aires, Argentina
}

neurite outgrowth and filopodium formation [2,3]. In addition, all PLP family members share a common structure, which includes two extracellular loops that potentially interact with external ligands, and four transmembrane domains. The PLP family is widely evolutionarily conserved from arthropods to mammals $[4,5]$. M6a, a membrane glycoprotein, is prominently expressed in the central nervous system, in particular in the hippocampus, cortex, forebrain, cerebellum, and retina $[1,6,7]$. Several lines of evidence showed M6a participation in neural development, such as neurite extension and/or filopodium/spine formation in hippocampal [3], retinal [8], and cerebellar [6] neurons, as well as in axonal growth [9]. Indeed, M6Ab, a zebra fish paralog of M6a, also exhibits similar functions [10]. M6a may also be required for filopodium motility and synaptogenesis $[8,11,12]$ and has been implicated in neuronal differentiation of human stem cells [13] and PC12 cells [14].

Chronic social and physical stress decreases Gpm6a mRNA levels in the hippocampus, and this downregulation is prevented by administration of antidepressants 
$[15,16]$, which suggests that M6a participates in plastic hippocampal changes observed in stressed/antidepressant-treated animals. However, the underlying mechanisms remain poorly understood. Interestingly, $M 6 b$ and DM20 are also regulated by chronic stress [2]. In contrast, PLP mRNA, a DM20 splice variant, which is abundantly expressed in myelin of the central nervous system, is not regulated by stress [2]. However, PLP participates in maintaining structural integrity of the myelin membrane. PLP and DM20 have also been shown to form a complex with integrins in oligodendrocytes [17].

Previous work depicting major steps in PLP evolution identified M6 as the ancestral gene of the PLP family present in Drosophila, maintaining a high degree of conservation in gene structure and amino acid sequence of the predicted protein compared with mouse M6a $[5,18,19]$, suggesting that M6 is the M6a fly ortholog. Therefore, the role of M6a was analyzed in Drosophila in the present study. In a previous study, we demonstrated that M6 localizes to the membrane of the ovary follicular epithelium, and M6 knockdown triggers female sterility [20]. Loss of M6 in follicle cells also impairs eggshell formation and epithelial integrity, as well as organization. Therefore, M6 plays an essential role in follicular epithelia maintenance, likely via membrane cell remodeling [20]. However, to date, there is no experimental evidence for M6 functional conservation, localization, or function in the fly nervous system.

To address the role of M6a in an intact nervous system, M6 relevance was characterized in adult flies. Results identified novel M6 isoforms that were differentially expressed in the ovaries and heads. All M6 isoforms were structurally and functionally conserved, with one exception; this isoform exhibited a different subcellular localization most likely due to an altered protein structure, thereby giving rise to a non-functional isoform. M6 localization was detected in several brain structures, most remarkably in the optic lobe neuropil. In addition, M6 mutant flies exhibited a defective response to light. These results identified M6 as one of the molecular components underlying phototactic behavior, and together with M6 localization in the optic lobe, results suggests that M6 might play a role in the fly visual system.

\section{Methods}

\section{Fly strains}

Flies were grown and maintained at $25^{\circ} \mathrm{C}$ under a $12 \mathrm{~h}$ light/dark (LD) cycle in vials containing standard cornmeal-agar medium. A $w^{1118}$ stock was used as the control $(w)$. Potential M6 mutant stocks $y^{1} w^{67 c 23}$; P $\left\{\right.$ EPgy2\}M6 ${ }^{\text {EY07032 }}, w^{1118} ; \mathrm{P}\{\mathrm{GT} 1\} \mathrm{M} 6^{\mathrm{BG} 00390}$ and $w^{1118}$; Mi\{ET1\}M6 ${ }^{\mathrm{MB02608}} / \mathrm{TM} 3, \mathrm{Sb}^{1} \mathrm{Ser}^{1}$ were obtained from the Bloomington Stock Center [21] and were renamed $M 6^{01}, M 6^{02}$ and $M 6^{03}$, respectively [20]. The CA06602 stock $\left(M 6^{\mathrm{GFP}}\right)$ was obtained from the GFP Protein Trap Database at the Carnegie Institution [22]. The $M 6^{01}$, $M 6^{O 2}$ and $M 6^{\mathrm{GFP}}$ strains were backcrossed several generations to $w^{1118}$ to minimize background effects. The original P-element (EY07032) from $M 6^{01}$ was removed with the transposase $(\Delta 2-3)$ and the reverted P-excised allele was kept as $M 6^{\Delta 01-\text { rev }}$ (for details see [20]).

\section{mRNA isolation, RT-PCR and quantitative real time} reverse transcription polymerase chain reaction (RT-qPCR) Heads and ovaries were dissected from well-fed young flies and homogenized in Trizol Reagent (Life Technologies, Carlsbad California, USA) to isolate total RNA according to manufacturer's instructions. Then, polyA+ mRNA was purified using the PolyATract mRNA Isolation System (Promega, Madison, WI, USA). Complementary DNA was synthesized using oligo dT and SuperScript ${ }^{\mathrm{TM}}$ II Reverse Transcriptase (Life Technologies, Carlsbad California, USA).

qPCRs were carried out in a 7500 Real-Time PCR System (Applied Biosystems, Foster City, California, USA). Quantitation of each cDNA was achieved using SYBR Green PCR Master Mix (Applied Biosystems) in triplicate. Primer sequences for housekeeping genes and M6 3'UTR were published elsewhere [20]. The oligonucleotide sequences used were: 5'AGAAATTCCAACGCAA CTAACAAA3' and 5'TGTTTCCAACTGGCAATGCA3', forward and reverse primers, respectively, for $M 6-A / C / D$ variants (P1, black arrows, Figure 1A); 5'TCACTGTGT GCCGTTTAGCTTG3' and 5'TTTATGGAGTCGAAGT CGGAATTT3' forward and reverse primers, respectively, for the M6-B variant (P2, black arrows, Figure 1A). Normalization was accomplished using $R p 49$ and gapdh as housekeeping genes and resulted in almost identical patterns. Relative quantification was performed using a comparative $C_{T}$ method [23,24]. Before each experiment, the calibration curves were validated. Samples whose curves amplified out of the calibrated dynamic range were eliminated. All procedures followed the manufacturer's instructions.

\section{M6 transcripts identification, topology and motif predictions}

PCR were done with GoTaq DNA polymerase according to the manufacturer's instructions (Promega, Madison, WI, USA). The oligonucleotide sequences used were: 5'AATTCCCAACGCAACTAACAAATTG3' and 5'CTG TAxCTCCAGCTCGTTCAGGTT3', forward and reverse primers for $M 6-A / C / D$ variants (gray arrows, Figure $1 \mathrm{~A}$ ); and 5'ATTCGTTGCTCGGTGGTTATTG3' and 5'C TAGAAGCGATCCTTCGA3', forward and reverse primers for $M 6-B$ (gray arrows, Figure 1A). The PCR amplicons were cloned into pGEM-T Vector System (Promega, Madison WI, USA) and sequenced. cDNA sequences 


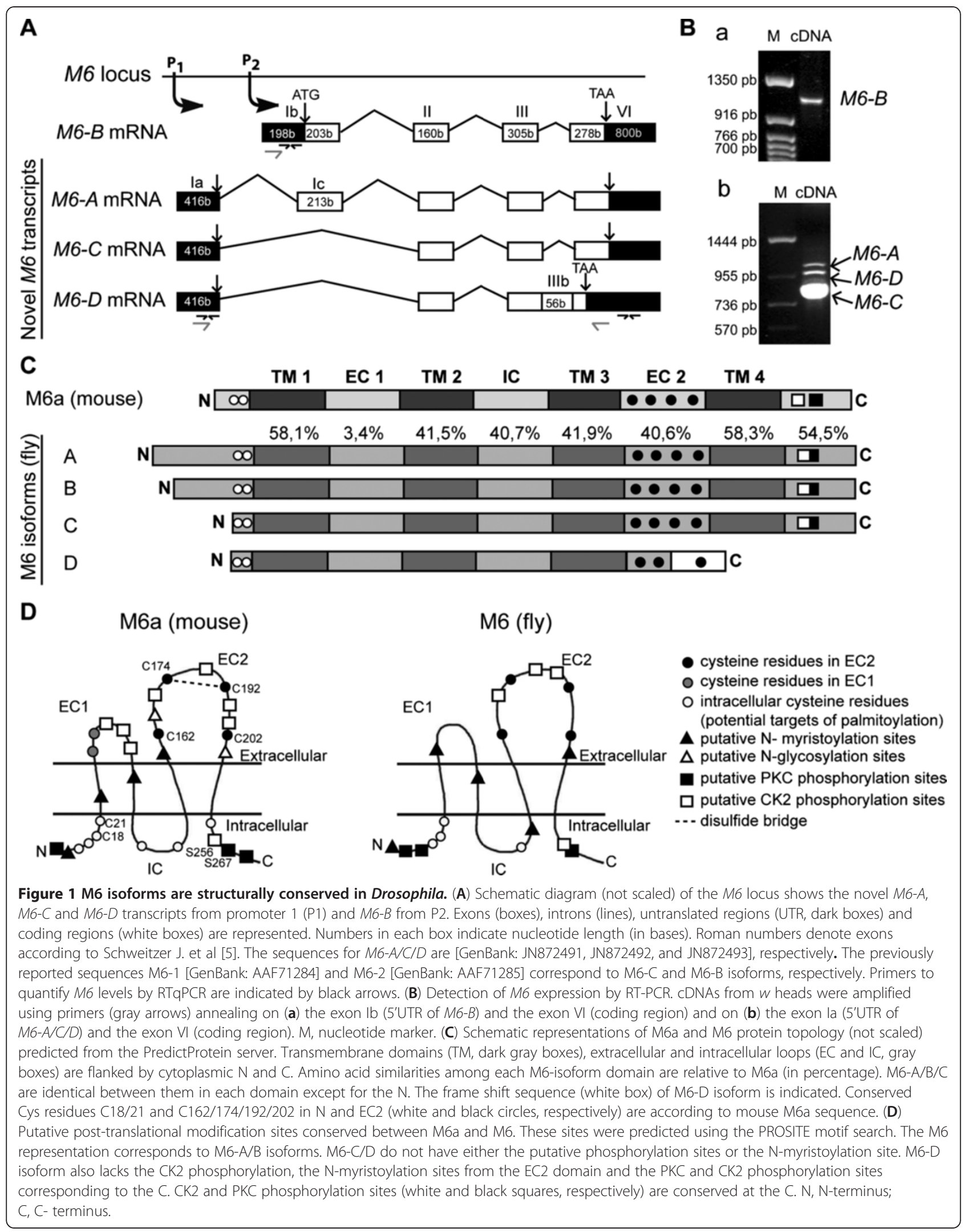


corresponding to novel M6 variants have been deposited in GenBank [25]. NCBI accession numbers are as follows: [GenBank: JN872491] (M6-A), [GenBank: JN872492] (M6-C) and [GenBank: JN872493] (M6-D). The forward and reverse primers annealing 38b downstream of the P1 site and 367b downstream of the stop codon were: 5'TTTTGAGCGAATTCAGTTGG3' and 5'GCATTCG GCAATTCAGAAGAA3, respectively.

Bioinformatic analysis included the Vector NTI Advance 10 software package, Kalign (2.0) and ClustalW (1.83) alignments [26]; the Ensembl website was used to determine exon/intron boundaries [27]. In addition, Predict Protein Server [28,29], which includes PHD predictions for protein topology, PROF predictions for motif scan (PROSITE) and DISULFIND for disulfide bridge prediction, were employed.

\section{Cloning and plasmids used}

To obtain GFP::M6-A, GFP::M6-B, GFP::M6-C and GFP::M6-D plasmids, all cDNAs were cloned into pGEM-T Vector System (Promega) and subcloned into pEGFP-C1 vector (BD Biosciences Clontech) with Pfu DNA polymerase (Promega) according to the manufacturer's recommendations. Primers were designed to amplify the complete coding sequences (CDS) from the initial ATG to the stop codon. Primers included the SacI and $K p n I$ restriction recognition sites to allow in-frame cloning into the pEGFP-C1 multiple cloning sites. Oligonucleotide sequences used were: 5'GAGCTCAAATGGC GTTGTGAAGTATG3', 5'GAGCTCAAAT GCCGGGCA AGGGGAACAA3' and 5'GAGCTCAAATGGGAGAAT GCTGCCAAT3', forward primers for $M 6-A, M 6-B$ and $M 6-C / D$, respectively; 5'GGTACCCTAGAAGCGATCC TTCGAGGT3' and 5'GGTACCTCATGTC CTCCAGTT TCGTGTT3,' reverse primers for $M 6-A / B / C$ and $M 6-D$, respectively. After the cloning step, plasmids were sequenced to exclude mutations. Control vectors, GFP:: M6a [12] expressed in the plasma membrane and GFP (GFP-PH, plasmid 21179, Addgene, Cambridge, MA, USA, [30]), which binds to plasma membrane phospholipds, were found to be enriched at the plasma membrane.

\section{Cell line and transfections}

Mouse neuroblastoma 2a (N2a, Clone CCL-131 ATCC, Manassas, VA, USA) cells were cultured in DMEM with 10$20 \%(\mathrm{v} / \mathrm{v})$ fetal bovine serum, penicillin, and streptomycin.

For transfections, we used polyethylenimine (PEI, School of Pharmacy and Biochemistry, UBA, Buenos Aires, Argentina). Briefly, $2 \mu \mathrm{g}$ of plasmid DNA and $3 \mu \mathrm{l}$ of $25 \mathrm{mM}$ PEI were diluted in $50 \mu \mathrm{l}$ of protein and antibiotic free medium (OPTI-MEM I Reduced Serum Medium, Gibco) and incubated for 8 minutes. Next, $200 \mu \mathrm{l}$ of complete medium were combined with transfection mix and added to each well in a 24 wells per plate format, containing cells previously washed twice with PBS (phosphate buffered saline). Cells were incubated with the transfection mix for 2 hours at $37^{\circ} \mathrm{C}$. Then, cells were washed 3 times with PBS and incubated with complete fresh medium.

\section{Cell staining and image analysis}

Twenty-four hours after transfection, N2a cells were fixed in $4 \%$ paraformaldehyde $/ 4 \%$ sucrose in PBS for 15 minutes at $4^{\circ} \mathrm{C}$. For F-actin staining, permeabilization was carried out with $0.1 \%$ Triton X-100 in PBS for 2 minutes. Cultures were blocked with 3\% BSA in PBS for 1 hour, followed by incubation with the rhodamine phalloidin 1/1,000 (Molecular Probes, Eugene, OR) in $3 \% \mathrm{BSA}$ in PBS at $37^{\circ} \mathrm{C}$ for $1 \mathrm{hr}$. Coverslips were incubated with DAPI and then were mounted with FluorSave Reagent (Calbiochem, La Jolla, CA). Fluorescent images were acquired by using a Nikon Eclipse 80i microscope (60x/1.4 objective) equipped with CoolLED pE excitation system and a confocal laser scanning microscope (Zeiss LSM510 Meta, 63x/1.4 objective).

The percentage of cells displaying filopodial protrusions (visualized by the F-actin marker phalloidin) was calculated for both transfected as well as nontransfected cells from the same coverslip. The percentage of non-transfected cells bearing filopodia from the same coverslip was used to normalize data. The ratio of transfected to non-transfected filopodium-bearing cells from the same coverslip was determined. A ratio similar to one implies that filopodium formation was not induced. At least 80 cells per coverslip were analyzed for 4 replicates from each experiment. Each experiment was independently repeated three times. Images were processed using Photoshop and Illustrator (Adobe Systems).

Whole brain immunohistochemistry and image analysis Adult brains from 3-6 day-old flies were dissected, fixed and stained as previously described [31]. Briefly, heads were fixed in $4 \%$ paraformaldehyde in $\mathrm{PB}(100 \mathrm{mM}$ $\mathrm{KH} 2 \mathrm{PO} 4 / \mathrm{Na} 2 \mathrm{HPO} 4)$ for 30 minutes to 1 hour at room temperature. The brain was dissected and washed with $0.3 \%$ Triton X-100 in PBS (PT). Brains were then blocked in 7\% goat serum in PT for 1 hour at room temperature and incubated with the primary antibody in PT (0.6\% Triton X-100) for 48 hours at $4^{\circ} \mathrm{C}$. Washes were carried out in PT $(0.6 \%$ Triton X-100) for 20 minutes and repeated twice prior to the addition of the secondary antibody. After a 2 hours incubation step, brains were washed for three times in PT (0.6\% Triton X-100), once in PT $(0.1 \%$ Triton X-100) and mounted in FluorSave Reagent (Calbiochem). All steps were carried out at room temperature unless otherwise indicated. The primary antibodies used were mouse anti-FasII (1D4, 1/5, 
Developmental Studies Hybridoma Bank [DSHB], IA, USA), anti-elav (9F8A9, 1/10, DSHB), and rabbit antiGFP (1/300, Molecular Probes, Invitrogen Carlsbad, CA USA). Secondary antibodies conjugated to $\mathrm{Cy} 2$ or $\mathrm{Cy} 3$ were used (1/500, Jackson ImmunoResearch Laboratories West Grove, PA, USA). Detection of GFP::M6 in the adult brain was repeated at least three times examining $8-10$ brains in each experiment. Brains from white flies were used as a negative control to confirm the specificity of the GFP antibody. Fluorescent images were acquired with the laser scanning confocal microscope Zeiss LSM510 Meta using 20x/0.8, 40x/1.3 and 63x/1.4 objectives. Images were processed using Photoshop and Illustrator (Adobe Systems).

\section{Lifespan analysis}

Survival was determined at $25^{\circ} \mathrm{C}$ under $\mathrm{LD}$ conditions. One hundred male flies from each genotype were maintained in vials (10 flies/vial) containing standard medium. Flies (0-48 hours old) were placed in vials and were scored for survivorship every 3-4 days, when they were transferred to fresh vials to minimize death caused by bacterial infection or moist in the medium. Three independent experiments were carried out. Survival curves represent the percentage of surviving flies as a function of time. For statistical analysis the mean life span of each strain was calculated as the time (in days) at which survival reached $50 \%$ of the starting population. In all experiments, only males were used because female life span is known to depend upon reproductive history [32].

\section{Environmental scanning electron microscopy (ESEM) of adult compound eyes}

Young adult male flies (3-6 days) were collected, anesthetized and immobilized on the ESEM mount using water-based colloidal carbon glue for proper orientation. The electroscan was performed with an environmental scanning electron microscope (ESEM, model XL30, Philips) at $20.0 \mathrm{kV}$ and 0.9 Torr in the auxiliary mode. This technology does not require metal coating of the specimen.

\section{Phototactic behavior}

Before each assay, 40 adult males of 2- to 3-days old were selected under $\mathrm{CO}_{2}$ and allowed to recover in fresh food vials for 1-3 days in LD. Phototactic behavior was performed as previously described [31]. Briefly, a horizontal device allows the "collecting tubes" to slide through the one containing the flies at the beginning of the experiment, which is always kept in the same position in reference to the light source. At least 15 minutes before testing flies were transferred to darkness for adaptation; further manipulations were performed under a safe red light. Flies were moved to a "test" tube $(13 \mathrm{~cm}$ long, $1 \mathrm{~cm}$ wide). Five "collecting" tubes were placed opposite to the test one. The white cold light source (150 Watt quartz halogen fiber optic illuminator, FiberLite MI-150) was initially placed right behind the collecting tube 1 , and kept in line with the test tube throughout the experiment. Each collecting tube was allowed to connect sequentially with the test tube for 1 minute. Thus, flies were allowed to freely move to the collecting illuminated tube for 1 minute, and then the tube was moved to the next position. The number of flies in each (collecting and test) tube was counted, and the proportion of flies that had a positive phototactic response (defined as those that moved towards the light within the first 2 minutes of initiating the test, or stayed in the first 2 collecting tubes) was analyzed. In each experiment, the results were the mean of the scores from 2 trials recorded from 40 flies per genotype. Each experiment was independently repeated 5 times.

\section{Total fly locomotor activity measurement}

Spontaneous fly locomotor activity of 2-3-days old adult males was monitored by recording infrared beam crossings in glass tubes $(6.5-\mathrm{cm}$ length, $3-\mathrm{mm}$ inside diameter) using a commercially available Drosophila activity monitoring system (TriKinetics, Waltham, MA). Individual activity was scored under LD conditions for 3 consecutive days. Total activity levels were determined as total counts per day displayed for each fly. Statistical analysis included a Kruskall-Wallis test. Data were obtained from at least three independent experiments; $\mathrm{n}=30$ flies per genotype in each experiment.

\section{Statistical data analysis}

Graphs were generated with GraphPad Prism software. Statistical analysis was performed with IS (Infostat software, Grupo InfoStat, FCA, Universidad Nacional de Córdoba, Argentina). Group means were analyzed for overall statistical significance by one-way analysis of variance (ANOVA) followed by multiple comparison tests. Non-parametric analysis was performed (Kruskal-Wallis followed by multiple comparison test) when assumptions on the normal distribution and variance did not allow otherwise.

\section{Results}

Novel M6 variants are generated by alternative splicing The M6 gene is located in 78D4 of chromosome $3 \mathrm{~L}$. The complete $M 6$ exon-intron structure comprises $4-5$ exons, which span a genomic interval of $4.9 \mathrm{~kb}$. Two depicted transcription initiation sites (P1 and P2) give rise to four M6 mRNA variants (Figure 1A) and four predicted proteins that contain 187 to 319 amino acids (aa).

Early in the study, only isoforms M6-A (CG7540-RA, [GenBank: NM141066]) and M6-B (CG7540-RB, 
[GenBank: NM168911]) had been reported as predicted transcript variants encoded by the M6 gene in the FlyBase [33], with both transcripts producing apparently the same protein, M6 A-B (314 aa). RT-PCR analysis of wild-type ovaries and heads with primers annealing to the end of isoforms $M 6-A$ or $M 6-B$ (gray arrows, Figure 1A) resulted in the identification of two novel shorter $M 6$ transcripts $(\sim 0.8 \mathrm{~kb}$, Figure $1 \mathrm{~B} \mathrm{~b})$, as well as the expected M6 transcripts $(\sim 1 \mathrm{~kb}$, Figure $1 \mathrm{~B}$ a). Sequence analysis indicated that shorter cDNAs were novel variants expressed in $D$. melanogaster. The novel M6 transcripts, termed M6-C [GenBank: JN872492] and M6-D [GenBank: JN872493], are two variants derived from alternative splicing. Both novel transcripts initiate at P1 and lack the first coding exon (exon Ic), thereby giving rise to an $\mathrm{N}$-terminus shorter than the one in M6-A (Figure 1A-C). The M6-C predicted protein has 248 aa. Previous work reported sequences for M6 in Drosophila ([GenBank: AF253528]; $[5,18,19])$ as M6-1 [GenBank: AAF71284] and M6-2
[GenBank: AAF71285]. Through sequence alignment, we determined that those sequences correspond to M6-C and M6-B isoforms, respectively, described in the present study (data not shown). In addition, M6-D has 56 additional bases (b) resulting from retention of the last intron (IIIb, Figure 1A), which shifts the reading frame and creates a premature stop codon located 183 bases upstream of the M6- $A$ stop codon. Therefore, this $M 6-D$ variant is predicted to give rise to a shorter protein (187 aa, Figure $1 \mathrm{C}$ ), with 31 novel aa at the C-terminus. In addition, there was a difference between the M6- $A$ predicted variant reported in the database (CG7540-RA; [GenBank: NM141066]) and the cloned variant from the present study [GenBank: JN872491]. The new variant has 15 additional base pairs, 10 of which are within the first coding exon (exon Ic). Therefore, the predicted protein has 319 aa, with an N-terminus slightly longer than M6-A reported in the database. Additional primers were used to confirm these results, suggesting that the novel variants
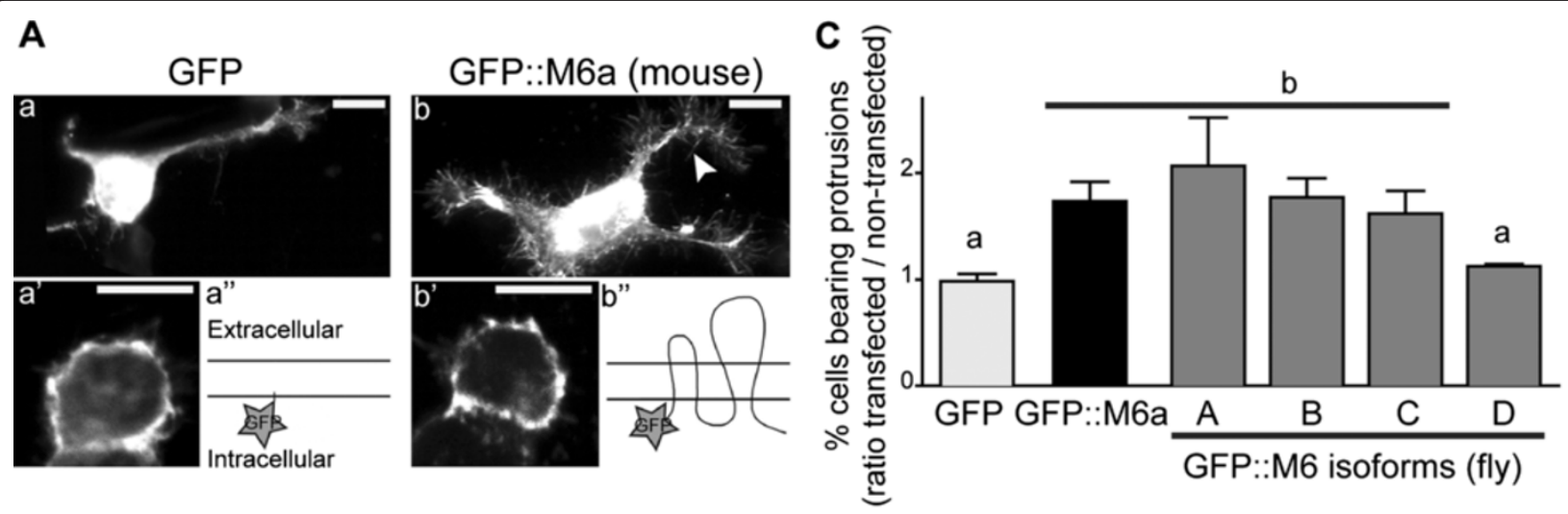

B

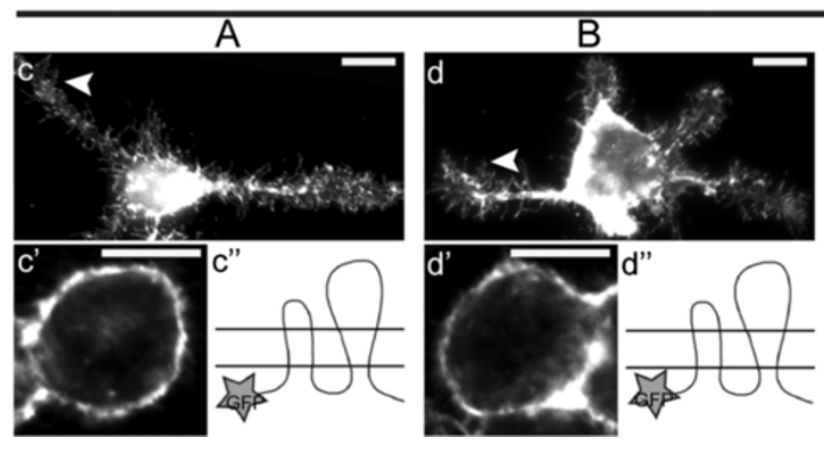

GFP::M6 isoforms (fly)

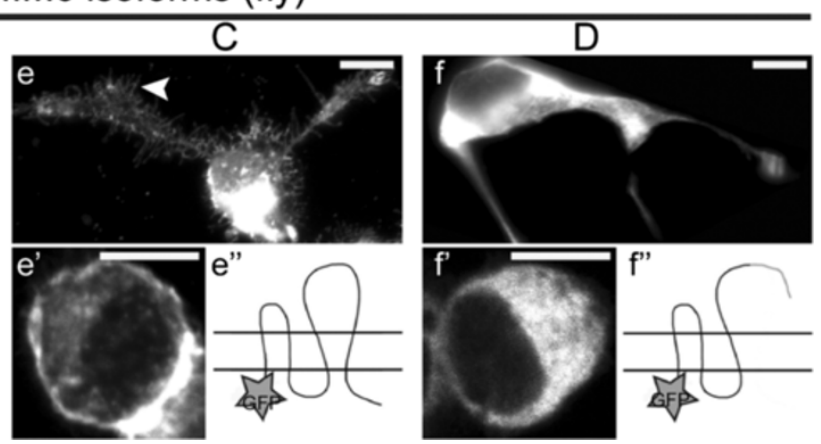

Figure 2 M6 isoforms are functionally conserved in Drosophila. Twenty-four hours after transfection N2a cells were fixed and stained with phalloidin to visualize F-actin and assess filopodium formation. (A-B) Fluorescent images of transfected N2a overexpressing GFP (a), GFP.:M6a (b) and the fruit fly GFP.:M6 isoforms (c-f). Arrowheads indicate filopodial protrusions. Confocal images showing the subcellular localization of GFP

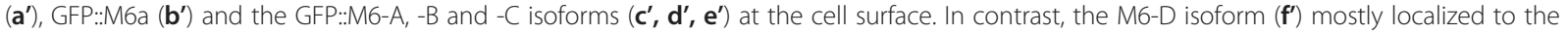
intracellular compartment. Scale bar is $10 \mu \mathrm{m}$. A schematic representation of the protein conformation $\left(\mathbf{a}^{\prime \prime}, \mathbf{b}^{\prime \prime}, \mathbf{c}^{\prime \prime}, \mathbf{d}^{\prime \prime}, \mathbf{e}^{\prime \prime}, \mathbf{f}^{\prime \prime}\right)$ is also included. $(\mathbf{C})$ M6 overexpression induced filopodium formation in N2a cells. The ratio of transfected to non-transfected filopodium-bearing cells from the same coverslip were determined. A ratio value similar to one implies that filopodium formation was not induced. Mean \pm SEM; $N=3$. Statistical analysis included a reciprocal data transformation (multiplicative inverse) and a Randomized Block ANOVA $(P<0.05)$ followed by a BBS multiple comparison. Different letters indicate significant differences. 
(M6-C and -D) contain the 5'UTR corresponding to the P1 promoter and share the same 3'UTR (data not shown).

\section{M6 is structurally conserved}

Using the 'Predict Protein' Server [29] and other bioinformatic tools, it was possible to predict the M6 tertiary structure. M6, mammalian M6a, and other PLP family members comprise four transmembrane domains (TM), a minor (EC1) and a major (EC2) extracellular loop, one intracellular loop (IC), and both $\mathrm{N}$ - and $\mathrm{C}$ - termini, which were localized within the cytoplasm. Figure $1 \mathrm{C}$ shows the comparison at the primary structure level between M6a (mouse) and M6 (fly) isoforms. The similarity percentage between different domains of M6a and M6 is also noted.

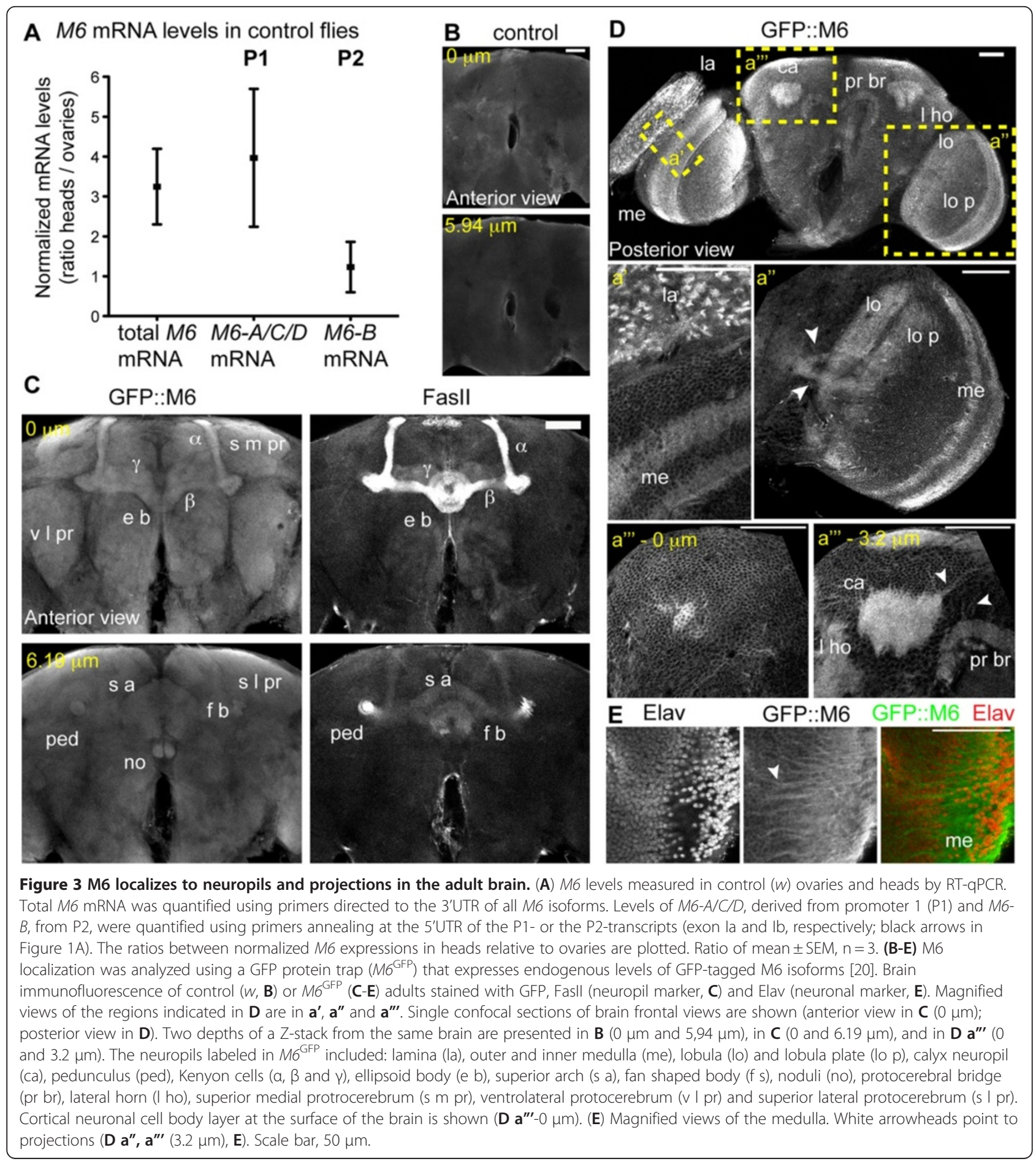


The TM domains, EC2, IC, and C-terminal regions share a high similarity (40-60\%) between mouse and fly sequences.

In addition, alignment of all M6 isoforms revealed that M6-A, -B, and -C are identical, with exception of the $\mathrm{N}$ terminal region. M6-A exhibits a longer $\mathrm{N}$-terminus than M6-B, whereas M6-C and -D display a shorter one. Variability at the $\mathrm{N}$-terminal region is due to alternative initiation sites (P1 and P2), as well as alternative splicing of the first coding exon (exon Ic, Figure 1A). In contrast, M6-D lacks the last transmembrane domain. Therefore, the C-terminus is localized outside the cell, which suggests that the major extracellular loop (EC2) does not adopt a proper conformation owing to the frame shift and premature stop codon.

We also sought for putative target sites for posttranslational modifications within the M6 sequence. The analysis revealed several conserved motives for phosphorylation (casein kinase 2 (CK2) and phosphokinase C (PKC)), Nmyristoylation, and key cysteine (Cys) residues. Interestingly, these sites are conserved in mammalian M6a. Predictive models of tertiary structures with sites conserved between mouse M6a and fly M6 are shown in Figure 1D. EC2 Cys residues forming disulfide bonds essential for M6a function [12], and two N-terminal Cys residues, which are conserved in all PLP family members of vertebrate and invertebrate organisms [5], are present in most M6 isoforms (black and white circles, respectively, Figure $1 C$-D). In particular, fly M6 and mouse M6a share three Cys residues at the intracellular N-terminus. In contrast, the M6-D isoform lacks the third Cys residue in EC2, and a fourth Cys residue appears in the frameshifted sequence. CK2 and PKC phosphorylation sites at the Cterminus (black and white squares, respectively, Figure 1 C-D) correspond with M6a phosphorylated S256 and S267 sites [34,35], which could be crucial for M6a function $[10,11,14]$.

\section{M6 is functionally conserved}

Because M6 isoforms are predicted to be transmembrane proteins (Figures 1C-D and 2 B c", d", e", f") and M6a is localized to the plasma membrane [12], localization of the various M6 variants was analyzed. M6 isoforms tagged to GFP were overexpressed in murine neuroblastoma 2a (N2a) cells, and localization was determined by confocal section analysis. Similar to mammalian M6a (Figure 2A b'), M6-A, $\mathrm{B}$, and $-\mathrm{C}$ exhibited cell surface expression (Figure 2B c'-e'). In contrast, M6-D, which lacks the fourth TM and EC2 domains, was mostly restricted to intracellular compartments (Figure 2B f').

Mouse M6a has been shown to induce filopodium/spine formation in neural and non-neural cells [3]. Therefore, to determine functional conservation of the distinct fly M6 variants, filopodium formation was analyzed. Briefly, N2a mammalian cells were transfected with fly M6 isoforms or mouse GFP-M6a, as well as GFP alone as the control (Figure 2A a-b, B c-f, $2 \mathrm{C}$ ). The percentage of cells bearing filopodial protrusions was quantified for transfected, as well as non-transfected cells through visualization of the F-actin marker phalloidin. The ratio of transfected to nontransfected filopodium-bearing cells was calculated to measure variant-specific overexpression resulting in filopodium formation. GFP overexpression did not induce filopodium formation (ratio = 1), whereas GFP::M6a overexpression exhibited significant induction (approximately 2-fold, Figure 2C). Overexpression of M6-A, -B and -C isoforms induced filopodium formation similar to mammalian M6a, which significantly varied from GFP alone $(P<0.05$, Figure $2 C)$. In contrast, M6-D, the truncated isoform lacking the major extracellular loop (EC2) and the fourth TM domain (Figure 2B f'), did not induce filopodium formation (Figure $2 \mathrm{C}$ ). These results suggest that M6-A, - B, and -C are functionally conserved variants. In addition, altered protein structure was likely responsible for M6-D subcellular delocalization and subsequent functional loss. These results suggest that the novel M6-D variant encodes a non-functional isoform.

\section{M6 isoforms are differentially expressed}

Because $M 6 a$ is highly expressed in the mammalian nervous system, M6 mRNA expression was analyzed in Drosophila heads. Using qPCR, we observed prominent expression in the heads compared with M6 ovary expression (Figure 3A). Subsequently, we specifically quantified mRNAs produced by either $\mathrm{P} 1$ or $\mathrm{P} 2$ promoters in wild-type heads and ovaries (Figure 3A) using primers that annealed to the corresponding 5'UTR (black arrows, Figure 1A). The $M 6-B$ transcript from P2 was expressed at similar levels in both samples. However, a predominance of P1-derived transcripts (M6- $A$, $C$ and $-D$ ) was detected in the heads. These results reveal tissue-specificity in the M6 promoters, where P1derived transcripts are most abundantly expressed in fly heads.

\section{M6 localizes to several brain structures and neural projections}

Antibodies specific to M6 were generated to determine whether M6 protein was expressed in the D. melanogaster nervous system. However, the results were inconclusive. Therefore, we used the fly line $M 6^{\mathrm{GFP}}$, which reports endogenous levels of M6. Our previous results demonstrated that $M 6$ mRNA levels in $M 6^{\mathrm{GFP}}$ are not reduced compared with control flies, and only P1-specific isoforms were tagged in frame to GFP at the N-terminus [20]. Immunofluorescence images of whole-mount brains of young adult flies are shown in Figure 3B-E. Distinct neuropils were identified with antibodies specific to FasII (Figure 3C). In addition, antibodies specific to Elav, a 


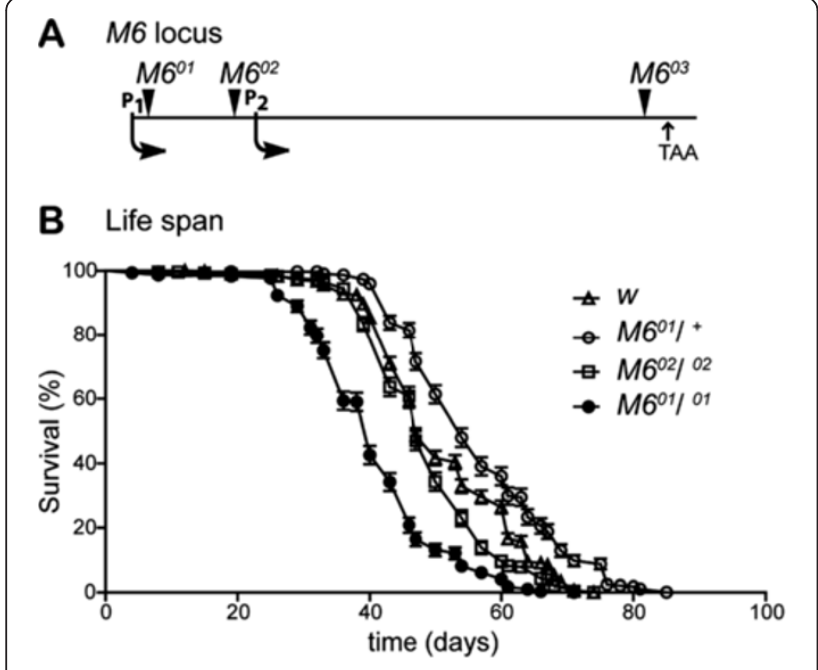

Figure 4 Median lifespan is reduced in M6 mutant flies. (A) Schematic diagram (not drawn to scale) of the M6 locus showing the localization of independent P-element insertions (arrowheads). Start transcription sites corresponding to P1 and P2 and the stop codon (TAA) are also indicated. (B) Survival curves of adult $\mathrm{W}, \mathrm{M}^{02}$ / ${ }^{02}$, heterozygous and homozygous $\mathrm{M}^{01}$ males $\left(\mathrm{MG}^{01} \mathrm{~J}^{+}\right.$and $\mathrm{M}^{01} \mathrm{\nu}^{01}$, respectively). The percentage of surviving flies \pm SE over time (in days) at $25^{\circ} \mathrm{C}$ in LD (standard conditions) is shown. Statistical analysis included a Log-rank (Mantel-Cox) survival curve comparison ( $P<0.0001$; Graphpad Software). Sample size ranged between 292 and 496 flies per genotype.

neuronal-specific marker, served as the counterstain (Figure 3E). In $M 6^{\mathrm{GFP}}$, a general staining of the major brain centers at the protocerebrum (comprising optic lobe, mushroom bodies, and central complex, Figure 3CD) was observed. In fact, GFP::M6 was expressed in most mushroom body structures (calyx neuropil, pedunculus, and Kenyon cells). In addition, GFP::M6 was expressed in the central body complex, more precisely in the ellipsoid body, superior arch, fan-shaped body, noduli, and the protocerebral bridge (Figure 3C). Other regions of the protocerebrum, such as the lateral horn, superior medial protocerebrum, ventrolateral protocerebrum, and superior lateral protocerebrum exhibited M6 expression (Figure 3C, D a"'-3.2 $\mu \mathrm{m}$ ).

In addition, neuropils most predominantly expressing GFP were present in the visual system (lamina, outer and inner medulla, lobula, and lobula plate) (Figure 3D). Interestingly, although entire neuropils were labeled, specific neural projections within the optic lobe that projected to the central brain, medulla, calyx, and protocerebral bridge were also labeled (arrowheads in Figure 3D a, a"'-3.2 $\mu \mathrm{m}$, 3E). GFP::M6 localization in several neuropils and in the cortical neuronal cell layer at the brain surface (Figure 3D a"'-0 $\mu \mathrm{m}$ ), as well as Elav localization, further supported M6 neuronal expression.

\section{M6 downregulation reduces lifespan}

To determine the role of M6 in Drosophila lifespan, potential M6 mutants containing inserted transposons within the M6 locus (Figure 4A) were characterized. $M 6^{01}$ flies contain a P-element inserted into the first exon (exon Ia), which corresponds to the 5'UTR of M6$A,-C$, and $-D$ transcripts, while $M 6^{02}$ contains a different P-element within the first intron. Our group previously demonstrated that $M 6^{01} /^{01}$ flies are hypomorphic mutants [20]. In the present study, the role of M6 in fly survival was evaluated under normal conditions. The lifespan of $M 6^{01}$ hypomorphic mutants was compared with that of homozygous $M 6^{02}$, heterozygous $M 6^{01}$, and $w$ (control) flies. The male flies did not exhibit any change in maximal lifespan (85 days for $M 6^{01} /^{+}, 71$ for $M 6^{01} /^{01}$, and 74 for $M 6^{02} /^{02}$ vs. 74 days for $w$, Figure 4B). However, when we examined the median lifespan, a parameter that indicates the time in which half of the population has died, significant differences were observed. While $M 6^{02} /{ }^{O 2}$ flies behaved similarly to control flies, a significantly reduced median lifespan was detected in homozygous $M 6^{01}$ males compared with both controls ( $40 \pm 3$ vs. $47 \pm 2$ and $54 \pm 3$ days for $M 6^{01}$ / o1, $w$ and $M 6^{01} /^{+}$flies, respectively; $\left.P<0.0001\right)$. Because the $M 6^{01}$ flies were backcrossed 10 times to $w$ flies to homogenize the genetic background, we conclude that the observed reduction in lifespan is indeed due to reduced $M 6$ expression.

This finding opens the provocative possibility that M6 might play a role in regulating animal aging (Figure 4B).

\section{Reduced M6 levels result in mild defects in the adult eye structure}

M6 expression was measured in fly heads of control and M6 mutants (Figure 5A). In addition to lines previously described, a transheterozygous $M 6^{01} /{ }^{03}$ line was included in the analysis. The $M 6^{O 3}$ line has a P-element insertion in the last coding exon (exon VI) of all functional variants and was determined to be homozygous lethal at the embryonic stage (Figure 4A). Heterozygous $M 6^{01}$ and $M 6^{02} \rho^{2}$ flies exhibited $M 6$ mRNA levels similar to the $w$ control. In contrast, homozygous $M 6^{01}$ and transheterozygous $M 6^{01} \rho^{03}$ flies exhibited significantly reduced M6 mRNA levels compared with control flies $(P<0.01$, Figure 5A). In addition, in the P-excised $M 6^{\Delta 01-r e v}$ flies, M6 was restored to normal levels, suggesting that the Pelement insertion in $M 6^{01}$ reduced $M 6$ expression in the heads.

Characterization of the $M 6$ mutants revealed morphological alterations in homozygous $M 6^{01}$ eyes, which suggested that M6 might play a role in this structure. To evaluate M6 participation in eye development, adult eyes were examined by environmental scanning electron microscopy (ESEM). The control flies, $w$ and 


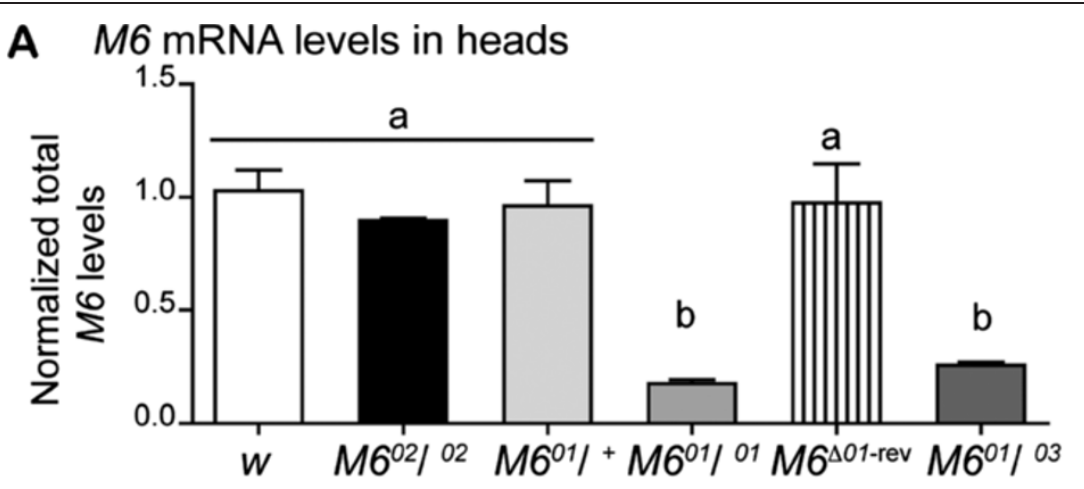

\section{B ESEM}
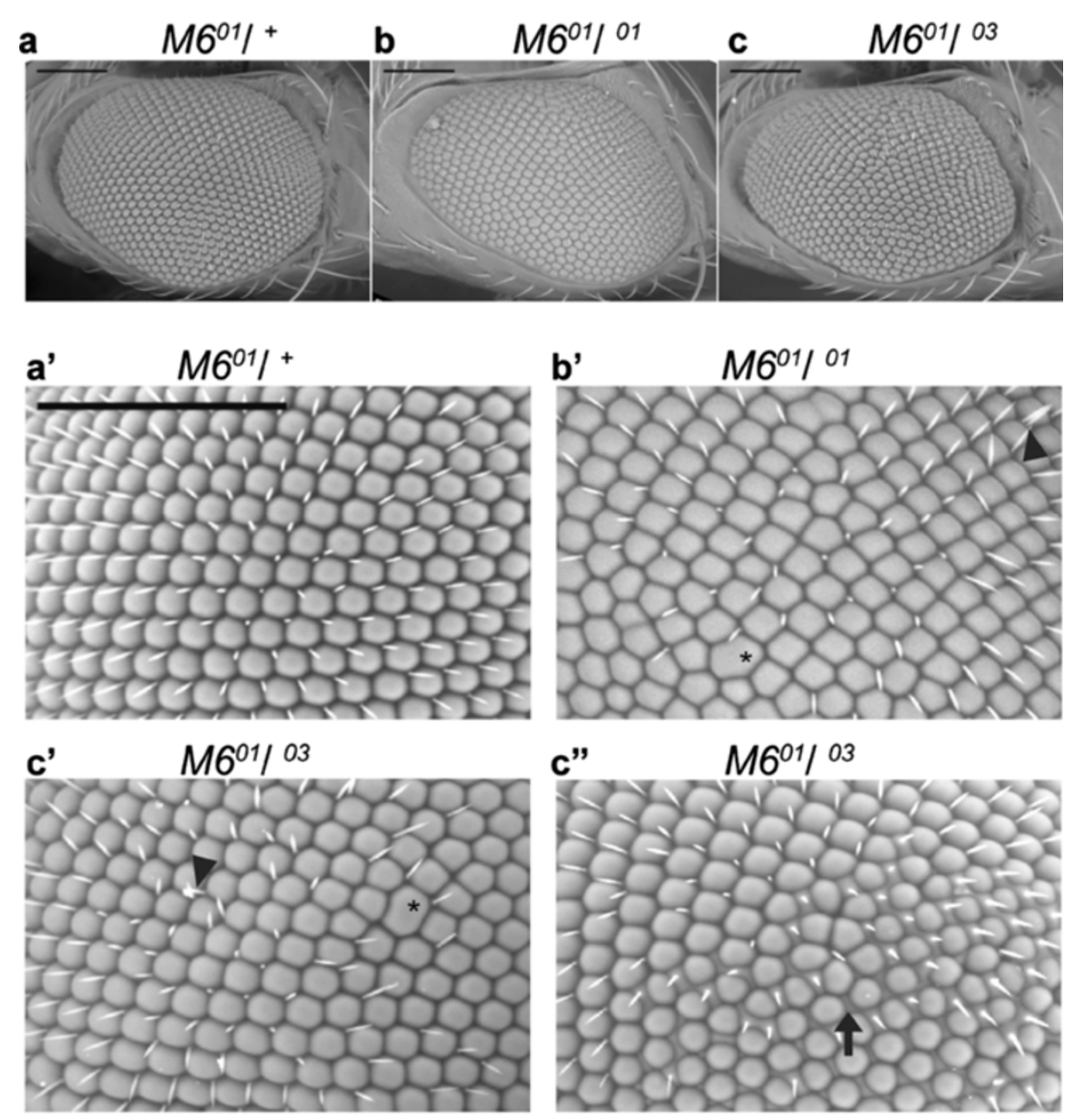

C Phototactic behavior

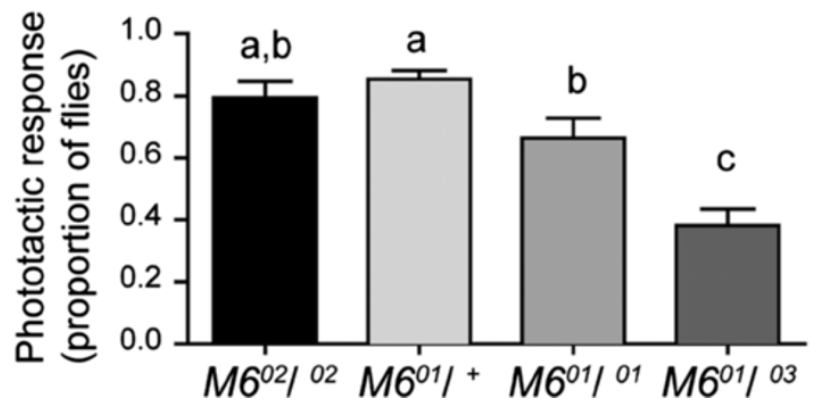


(See figure on previous page.)

Figure 5 M6 downregulation triggers a mild rough eye phenotype and impairs the phototactic response. (A) M6 mRNA levels in heads of

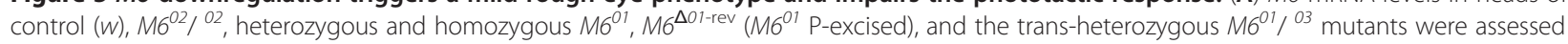
by RT-qPCR. The values shown are relative to the control genotype (white bar). Mean $\pm S E M, n=3-5$. Kruskal-Wallis test, $P<0.001$, followed by a multiple comparison test. Different letters indicate significant differences. (B) Representative images of young male fly eyes taken with an environmental scanning electron microscope (ESEM). Eyes of heterozygous $\mathrm{MG}^{01} /^{+}$used as control (a, $\mathbf{a}^{\prime}$ ), homozygous $\mathrm{Mb}^{01} /{ }^{01}$ (b, $\mathbf{b}^{\prime}$ ) and transheterozygous $\mathrm{M}^{01} \mathrm{I}^{03}$ (c, $\mathbf{c}^{\prime}$ and $\mathbf{c}^{\prime \prime}$ ) are shown. Lower and higher magnifications are displayed in the upper and lower panels, respectively. In addition to the disorganized ommatidium array, alterations in the ommatidial shape or fusions between contiguous ommatidia (asterisks) and bristle defects (arrowheads) are indicated. Altered interommadial space was detected in around $20 \%$ of $\mathrm{M6}^{01} /{ }^{03}$ mutants (arrow, $\mathbf{c}^{\prime \prime}$ ). The ventraldorsal axis is oriented left to right; scale bar is $100 \mu \mathrm{m}$. (C) Young male flies were assessed for their response to light in the phototaxis behavioral paradigm. Mean $\pm \mathrm{SEM}, \mathrm{n}=5$ independent experiments. Statistical analysis included a two way ANOVA $(P<0.0001)$ followed by a Newman-Keuls multiple comparison test between genotypes.

heterozygous $M 6^{01}$, exhibited a stereotypical, uniform, ommatidium pattern (Figure 5B a, and data not shown). In contrast, in the $M 6$ hypomorphic mutants $\left(M 6^{01}{ }^{01}\right.$ and $M 6^{01} \rho^{33}$ ), a mild rough-eye phenotype was observed, which was composed of a disorganized ommatidium array, defects in ommatidium shape and/or ommatidium fusion (asterisks in Figure 5B b', c'), and defective or missing bristles (arrowheads). In addition, approximately $20 \%$ of $M 6^{01} \rho^{33}$ transheterozygote eyes exhibited an excessive interommatidial space (arrow in Figure 5B c"). These results suggest that M6 plays a role during eye development.

\section{M6 is required for light response}

M6 is prominently expressed in the optic lobe, which suggests that M6 might play a role in the visual system, in particular in response to light. Therefore, a phototaxis paradigm was used to assess detection and processing of light information. Wild-type adult flies exhibit positive phototactic behavior. Because $w$ flies do not have pigmented eyes, the $M 6^{02} \rho^{2}$ and $M 6^{01} /^{+}$flies were used as controls to account for the potential contribution of eye pigmentation. Although there were subtle differences between $M 6^{01} \rho^{01}$ phototactic responses, the $M 6^{01} \rho^{01}$ and $M 6^{01}{ }^{33}$ young males exhibited a significantly defective response compared with controls $(P<0.0001$, Figure $5 C)$, thereby suggesting that $M 6$ is required for normal responses to light. Although $M 6$ mRNA levels were similarly reduced in $M 6^{01} \rho^{01}$ and $M 6^{01} \rho^{03}$ mutants, the phenotypic consequences were greater in $M 6^{01} \rho^{03}$. Although the exact nature of this difference remains to be determined, it is worth mentioning that the P-element in $M 6^{01}$ only affects a subset of splice variants $(M 6-\mathrm{A} / \mathrm{C} / \mathrm{D}$, unpublished data), while the P-element in $M 6^{03}$ is inserted in a region common to all functional variants (M6-A/C and M6-B), likely affecting all isoforms. Our results suggest that the differences in phototactic response could be a result of differential M6 variant expression in each specific mutant.

To rule out that the defective response to light induced by $M 6$ downregulation was due to a general decrease in locomotor activity, adult M6 mutant flies were analyzed in Drosophila activity monitors (Trikinetics, Walthman, MA). Interestingly, $M 6^{01} \rho^{01}$ and $M 6^{01}{ }^{33}$ males did not exhibit significant differences compared with control flies $\left(w, M 6^{02}\right.$, or $M 6^{01} /^{+} ; P>0.05, \mathrm{n}=3$ independent experiments, data not shown). Therefore, the impaired light responsiveness was more likely derived from higher-order visual processing defects triggered by M6 downregulation.

\section{Discussion}

Results from the present study demonstrate that Drosophila M6 is expressed in the fly nervous system. In addition, we identified two novel isoforms and further described the organization of the M6 gene. The expression of most isoforms in neuroblastoma cells resulted in protrusions similar to those previously reported for mouse M6a, which suggests that M6 constitute the functional ortholog of mammalian M6a. In addition, analyses of insertional mutants demonstrated that M6 is required for a proper adult phototactic response.

\section{Structural conservation of PLP family members}

The generation of transcript variants by distinct promoters and alternative splicing seems to be a common feature in PLP family member genes conserved through evolution. Similar to Drosophila M6 organization, zebrafish $D M \beta 2$ and rat gpm6a (isoforms Ia and Ib) exhibit 5'UTR alternative splicing that produces two N-termini [5,36]. The human GPM6A gene exhibits three transcripts [GenBank: NM005277, NM201591, NM201592] that differ at the N-terminus owing to specific transcriptional start sites and/or alternative splicing in the first coding exon. Similar features have been described for other members of the PLP family, such as $m 6 b$ and $p l p-1$ [19]. In the present molecular analysis of Drosophila M6, we have identified two novel M6 isoforms with a short $\mathrm{N}$-terminus (M6-C and -D), as well as a third isoform (M6-A) with a longer $\mathrm{N}$-terminus compared with those reported in the database. The M6 isoform variation at the $\mathrm{N}$-terminus is also due to alternative transcription start sites and alternative splicing of the first coding exon (exon Ic). Since M6 is the only PLP family member in 
Drosophila, we propose that the splice variants may tailor M6 according to specific needs of each tissue. In the case of Drosophila, we demonstrated that isoform expression is tissue-regulated by differential promoter activity (P1 in heads vs. ovaries, Figure 3). Similarly, Cooper et al. (2009) demonstrated tissue-regulated gpm6a expression in the rat; Ia and Ib variants from alternative promoters are differentially expressed in the brain and kidney.

\section{Functional conservation of PLP family members}

Results from the present study demonstrate that M6-A, $-\mathrm{B}$, and $-\mathrm{C}$ proteins localize to the cell surface in neuroblastoma cells. As previously shown for mouse M6a [3], all of them induced filopodium formation, demonstrating that M6 and M6a are indeed functional homologs. Interestingly, we also detected a non-functional isoform (M6-D) that was mostly restricted to the cytoplasm. M6D lacks the third Cys residue from the EC2 domain, as well as the fourth TM domain. Previous work from our group on the mouse M6a shows that the first and fourth Cys residues (C162 and C202) are crucial for cell surface expression and for M6a function [12]. In the case of fly M6-D, the absence of the last TM may prevent proper EC2 folding, resulting in a defective interaction between the first and fourth Cys, thus leading to altered subcellular localization and impaired filopodium formation. Consistent with Fuchsova et al. (2009), the EC2 domain structure appeared to be necessary for proper protein localization and function. In addition, PLP mutants lacking Cys residues from EC2 are misfolded and, therefore, are retained in the endoplasmic reticulum [27]. Interestingly, because M6-D is endogenously expressed in ovaries and heads (Figure 1B), it is possible that it might act as an endogenous regulator. Intriguingly, M6 expression is regulated by nonsense-mediated mRNA decay (NMD), which is a post-transcriptional regulation mechanism that targets transcripts containing early stop codons [37]. The $M 6-A$ transcript is an NMD-target, whereas $M 6-\mathrm{B}$ is not [37]. Because $M 6-A$ and $M 6-D$ transcripts share the 5 'UTR sequence, it is possible that $M 6-D$ also undergoes degradation via the NMD pathway.

\section{M6 functions in the visual system}

M6 was predominantly expressed in the fly adult optic lobe (Figure 3). In the mouse, M6a is present in neuronal processes of the retina, including axons of retinal ganglion cells during development, and inner and outer plexiform layers of adult retina. In addition, M6a overexpression in retinal cells enhances neurite outgrowth in vitro [8]. In Xenopus and zebrafish, M6a is expressed in the retina, in particular in the inner nuclear layer and ganglion cell layer [5,38]. Considering that the vertebrate and fly visual system share structural and functional molecular mechanisms, as well as developmental features [39], we conclude that M6a/M6/ $\mathrm{DMb}$ localization in the visual system is conserved through evolution.

Despite previous reports of retinal M6a expression [5,8,38], in vivo M6a function in the visual system has not been described. The present study provides the first experimental evidence for the requirement of M6 in the adult response to light (Figure 5C). Interestingly, although $M 6$ hypomorphic mutations resulted in a subtly altered eye structure in the adult (Figure 5B), M6 mutants exhibited very poor behavioral performance in a simple paradigm. These results suggest that M6 might play a role in higherorder visual processing, because structural alterations do not account for the defective light response. Indeed, immunofluorescence analysis of chaoptin localization (a well characterized marker of fly photoreceptors) in M6 mutant retinas did not exhibit any clear defect (data not shown). However, this observation does not preclude a role for M6 in retinal morphogenesis. In fact, directing M6-RNAi expression to the eye (employing ey-GAL4 and GMRGAL4) resulted in clear structural defects (data not shown). These results suggest a functional role for M6 in the establishment of the neural circuitry underlying visual processing.

The compound eye develops from a single-layered epithelium, the eye imaginal disc. Pupal eye development involves a coordinated series of morphogenetic events, such as cell-cell communication, differential cell adhesion, maintenance of cell polarity, cell shape, local cell movement, and programmed cell death, to properly pattern the ommatidia in the adult eye [40,41]. Accordingly, we previously reported a role for M6 in maintenance of the follicular epithelia, likely via cell adhesion, during cell remodeling [20], which was further supported by the observation of abnormal DE-cadherin distribution in the follicular epithelia of late egg chambers in M6 hypomorphic mutants (MPZ and MFC, unpublished data). Beta-integrin (mys) and M6 genetically interacted, which was supported by fly lethality when $M 6^{03}$ and $m y s^{1}$ alleles were combined (MPZ and MFC, unpublished data). Recently, our group demonstrated M6a localization in membrane microdomains, which are compatible with lipid rafts in primary hippocampal neuronal cultures [42]. Interestingly, misexpression of the Reggie/Flotilin lipid raft markers in the fly eye imaginal disc results in severe disturbance of the ommatidial pattern and specific and severe mislocalization of cell adhesion molecules [43]. These results suggest a role for M6 in cell-adhesion during eye development.

According to differential tissue expression of transcripts in flies, each variant might play distinct roles in different tissues. Therefore, M6 might play a dual role in cell remodeling in the visual system (present study) and epithelia [20]. 


\section{M6 functions in Drosophila}

In addition to the requirements of M6 during fly development [20], we demonstrated the role of M6 in adult survival. Because the median life span of hypomorphic $M 6^{01}$ males was slightly reduced, results suggest that M6 could also play a role in the regulation of animal aging.

M6 is expressed in several fly neuropils (Figure 3), including the central complex, a region involved in control of fly locomotion $[44,45]$. In addition, preliminary results showed that overall locomotor activity was slightly, although significantly, reduced (approximately $20 \%$, data not shown) in adult flies with silenced M6 expression specifically in the ellipsoid body (a central-complex structure) via a specific driver (c232-GAL4, [46]), suggesting that M6 could be required in neural circuits underlying this behavior. Notably, PLP mutant mice exhibit deficits in locomotor activity [47]. Our results also showed M6 expression in mushroom bodies. The mushroom bodies are analogous to the mammalian hippocampus, where M6a is abundantly expressed and regulated by chronic stress [3]. In flies, the mushroom bodies are crucial for olfactory learning and memory. Therefore, future studies should evaluate the role of M6 in this complex behavior.

Similarly, it has been reported that M6a expression in the adult brain is stronger in non-myelinated axonal fibers compared with myelinated axons [7]. Because proteolipid genes appeared earlier in evolution than myelin, it has been hypothesized that their involvement in myelination was acquired later $[4,5]$. Consistent with this, flies do not carry myelinated axons. In flies, nerve ensheathment depends on axonal insulation by glial cells, as well as the subsequent establishment of septate junctions between glial cell membranes. It is worth mentioning that cell junction organization and function share common features in vertebrates and invertebrates [48]. Therefore, M6a/M6 could potentially act as a mediator of cell-cell interactions involved in axon fasciculation during development. This possibility is also supported by the observation that in the Drosophila embryo, M6 co-localizes with Fasciclin II, a marker of longitudinal axon fascicles in the nervous system [20].

\section{Conclusions}

In conclusion, we have revealed tissue-differential expression of novel M6 isoforms, one of which was nonfunctional. $M 6$ was shown to be the functional fly ortholog of mouse Gpm6a. In addition, a role for M6 in the regulation of life span and in the in vivo fly visual system was revealed, which is particularly relevant owing to conservation of this protein between flies and mammals.

\section{Abbreviations}

CNS: Central nervous system; Cys: Cysteine; EC: Extracellular; ESEM: Environmental scanning electron microscope; GFP: Green fluorescent protein; Gpm6a: Glycoprotein M6a; IC: Intracellular; NMD: Nonsense-mediated
mRNA decay; PCR: Product chain reaction; PLP: Proteolipid protein; qPCR: Quantitative PCR; TM: Transmembrane; UTR: Untranslated region.

\section{Competing interests}

The authors of this manuscript do not have competing financial or nonfinancial interests in relation to this work.

\section{Authors' contributions}

MPZ conceived, designed, carried out the experiments, analyzed the data, performed the statistical analysis, and wrote the paper. SCB carried out the molecular and cellular studies. GB carried out immunohistochemistry on adult retinas. ACF conceived the study, analyzed the results, and helped to draft the manuscript. MFC conceived the fly's experiments, participated in their design, analyzed the results, and helped to draft the manuscript. MAB coordinated the study, helped to design the molecular and cellular experiments, analyzed the results and wrote the paper. All authors read and approved the final manuscript.

\section{Acknowledgments and funding}

We specially thank C. Rezával for immunohistochemistry experiments. We are grateful to the Drosophila Studies Hybridoma Bank for antibodies. We thank the Bloomington Drosophila Stock Center [21] and the GFP Protein Trap Database [49] for fly strains and the use of Fly base [33], NCBI database [25], EMBL-EBI tools [26] and Ensembl [50] databases.

This work was supported in part by the International Research Scholar from the $\mathrm{HHMI}$ to ACF, and grants from the Agencia Nacional para la Promoción Científica y Tecnológica (ANPCyT, PICT 2007-678 to MFC and PICT 2005-38204 to ACF). MPZ was supported by graduate fellowships from the National Council for Research in Science and Technology (CONICET) and UNSAM, GB was supported by CONICET, and MFC, MAB and ACF are members of CONICET.

Received: 1 December 2011 Accepted: 26 June 2012 Published: 4 July 2012

\section{References}

1. Yan $Y$, Narayanan $V$, Lagenaur C: Expression of members of the proteolipid protein gene family in the developing murine central nervous system.

J Comp Neurol 1996, 370:465-478

2. Fernandez ME, Alfonso J, Brocco MA, Frasch AC: Conserved cellular function and stress-mediated regulation among members of the proteolipid protein family. J Neurosci Res 2010, 88:1298-1308.

3. Alfonso J, Fernandez ME, Cooper B, Flugge G, Frasch AC: The stress-regulated protein M6a is a key modulator for neurite outgrowth and filopodium/ spine formation. Proc Natl Acad Sci U S A 2005, 102:17196-17201.

4. Mobius W, Patzig J, Nave KA, Werner HB: Phylogeny of proteolipid proteins: divergence, constraints, and the evolution of novel functions in myelination and neuroprotection. Neuron Glia Biol 2008, 4:111-127.

5. Schweitzer J, Becker T, Schachner M, Nave KA, Werner H: Evolution of myelin proteolipid proteins: gene duplication in teleosts and expression pattern divergence. Mol Cell Neurosci 2006, 31:161-177.

6. Lagenaur C, Kunemund V, Fischer G, Fushiki S, Schachner M: Monoclonal M6 antibody interferes with neurite extension of cultured neurons. J Neurobiol 1992, 23:71-88.

7. Cooper B, Werner HB, Flugge G: Glycoprotein M6a is present in glutamatergic axons in adult rat forebrain and cerebellum. Brain Res 2008, 1197:1-12.

8. Zhao J, lida A, Ouchi Y, Satoh S, Watanabe S: M6a is expressed in the murine neural retina and regulates neurite extension. Mol Vis 2008, 14:1623-1630.

9. Sato Y, Mita S, Fukushima N, Fujisawa H, Saga Y, Hirata T: Induction of axon growth arrest without growth cone collapse through the N-terminal region of four-transmembrane glycoprotein M6a. Dev Neurobiol 2011, 71:733-746

10. Huang KY, Chen GD, Cheng CH, Liao KY, Hung CC, Chang GD, Hwang PP, Lin SY, Tsai MC, Khoo KH, et al: Phosphorylation of the Zebrafish M6Ab at Serine 263 Contributes to Filopodium Formation in PC12 Cells and Neurite Outgrowth in Zebrafish Embryos. PLoS One 2011, 6:e26461. 
11. Brocco MA, Fernandez ME, Frasch AC: Filopodial protrusions induced by glycoprotein M6a exhibit high motility and aids synapse formation. Eur J Neurosci 2010, 31:195-202.

12. Fuchsova B, Fernandez ME, Alfonso J, Frasch AC: Cysteine residues in the large extracellular loop (EC2) are essential for the function of the stress-regulated glycoprotein M6a. J Biol Chem 2009, 284:32075-32088.

13. Michibata H, Okuno T, Konishi N, Kyono K, Wakimoto K, Aoki K, Kondo Y, Takata K, Kitamura Y, Taniguchi T: Human GPM6A is associated with differentiation and neuronal migration of neurons derived from human embryonic stem cells. Stem Cells Dev 2009, 18:629-639.

14. Mukobata S, Hibino T, Sugiyama A, Urano $Y$, Inatomi A, Kanai $Y$, Endo $H$, Tashiro F: M6a acts as a nerve growth factor-gated $\mathrm{Ca}(2+)$ channel in neuronal differentiation. Biochem Biophys Res Commun 2002, 297:722-728.

15. Alfonso J, Aguero F, Sanchez DO, Flugge G, Fuchs E, Frasch AC, Pollevick GD: Gene expression analysis in the hippocampal formation of tree shrews chronically treated with cortisol. J Neurosci Res 2004, 78:702-710.

16. Alfonso J, Frick LR, Silberman DM, Palumbo ML, Genaro AM, Frasch AC: Regulation of hippocampal gene expression is conserved in two species subjected to different stressors and antidepressant treatments. Biol Psychiatry 2006, 59:244-251.

17. Gudz TI, Schneider TE, Haas TA, Macklin WB: Myelin proteolipid protein forms a complex with integrins and may participate in integrin receptor signaling in oligodendrocytes. J Neurosci 2002, 22:7398-7407.

18. Stecca B, Southwood CM, Gragerov A, Kelley KA, Friedrich VL Jr, Gow A: The evolution of lipophilin genes from invertebrates to tetrapods: DM-20 cannot replace proteolipid protein in CNS myelin. J Neurosci 2000, 20:4002-4010.

19. Werner H, Dimou L, Klugmann M, Pfeiffer S, Nave KA: Multiple splice isoforms of proteolipid M6B in neurons and oligodendrocytes. Mol Cell Neurosci 2001, 18:593-605.

20. Zappia MP, Brocco MA, Billi SC, Frasch AC, Ceriani MF: M6 membrane protein plays an essential role in Drosophila oogenesis. PLoS One 2011, 6:e19715.

21. Bloomington Drosophila Stock Center:: http://flystocks.bio.indiana.edu/.

22. Buszczak M, Paterno S, Lighthouse D, Bachman J, Planck J, Owen S, Skora AD, Nystul TG, Ohlstein B, Allen A, et al: The carnegie protein trap library: a versatile tool for Drosophila developmental studies. Genetics 2007, 175:1505-1531.

23. Biosystems A: Guide to Performing Relative Quantitation of Gene Expression Using Real-Time Quantitative PCR. 2004.

24. Pfaffl MW: A new mathematical model for relative quantification in real-time RT-PCR. Nucleic Acids Res 2001, 29:e45.

25. NCBI. http://www.ncbi.nlm.nih.gov/.

26. EMBL-EBI. http://www.ebi.ac.uk/Tools/.

27. Dhaunchak AS, Nave KA: A common mechanism of PLP/DM20 misfolding causes cysteine-mediated endoplasmic reticulum retention in oligodendrocytes and Pelizaeus-Merzbacher disease. Proc Natl Acad Sci U S A 2007, 104:17813-17818.

28. PredictProtein Server. www.predictprotein.org/.

29. Rost B, Yachdav G, Liu J: The PredictProtein server. Nucleic Acids Res 2004, 32:W321-W326

30. Stauffer TP, Ahn S, Meyer T: Receptor-induced transient reduction in plasma membrane Ptdlns(4,5)P2 concentration monitored in living cells. Curr Biol 1998, 8:343-346.

31. Rezaval C, Berni J, Gorostiza EA, Werbajh S, Fagilde MM, Fernandez MP Beckwith EJ, Aranovich EJ, Sabio y Garcia CA, Ceriani MF: A functional misexpression screen uncovers a role for enabled in progressive neurodegeneration. PLoS One 2008, 3:e3332.

32. Luckinbill LS, Clare MJ: Selection for life span in Drosophila melanogaster. Heredity 1985, 55(Pt 1):9-18.

33. Flybase. http://flybase.org/.

34. Huttlin EL, Jedrychowski MP, Elias JE, Goswami T, Rad R, Beausoleil SA, Villen J, Haas W, Sowa ME, Gygi SP: A tissue-specific atlas of mouse protein phosphorylation and expression. Cell 2010, 143:1174-1189.

35. DeGiorgis JA, Jaffe H, Moreira JE, Carlotti CG Jr, Leite JP, Pant HC, DosemeC A: Phosphoproteomic analysis of synaptosomes from human cerebral cortex. J Proteome Res 2005, 4:306-315.

36. Cooper B, Fuchs E, Flugge G: Expression of the axonal membrane glycoprotein M6a is regulated by chronic stress. PLoS One 2009, 4:e3659.

37. Hansen KD, Lareau LF, Blanchette M, Green RE, Meng Q, Rehwinkel J, Gallusser FL, Izaurralde E, Rio DC, Dudoit S, Brenner SE: Genome-wide identification of alternative splice forms down-regulated by nonsensemediated mRNA decay in Drosophila. PLoS Genet 2009, 5:e1000525.

38. Yoshida M, Shan WS, Colman DR: Conserved and divergent expression patterns of the proteolipid protein gene family in the amphibian central nervous system. J Neurosci Res 1999, 57:13-22.

39. Sanes JR, Zipursky SL: Design principles of insect and vertebrate visual systems. Neuron 2010, 66:15-36.

40. Carthew RW: Pattern formation in the Drosophila eye. Curr Opin Genet Dev 2007, 17:309-313.

41. Cagan R: Principles of Drosophila eye differentiation. Curr Top Dev Biol 2009, 89:115-135.

42. Scorticati C, Formoso K, Frasch AC: Neuronal glycoprotein M6a induces filopodia formation via association with cholesterol-rich lipid rafts. J Neurochem 2011, 119:521-531.

43. Hoehne M, de Couet HG, Stuermer CA, Fischbach KF: Loss- and gain-offunction analysis of the lipid raft proteins Reggie/Flotillin in Drosophila: they are posttranslationally regulated, and misexpression interferes with wing and eye development. Mol Cell Neurosci 2005, 30:326-338.

44. Martin JR, Raabe T, Heisenberg M: Central complex substructures are required for the maintenance of locomotor activity in Drosophila melanogaster. J Comp Physiol A 1999, 185:277-288.

45. Strauss R, Heisenberg M: A higher control center of locomotor behavior in the Drosophila brain. J Neurosci 1993, 13:1852-1861.

46. Renn SC, Armstrong JD, Yang M, Wang Z, An X, Kaiser K, Taghert PH: Genetic analysis of the Drosophila ellipsoid body neuropil: organization and development of the central complex. J Neurobiol 1999, 41:189-207.

47. Boison D, Bussow H, D'Urso D, Muller HW, Stoffel W: Adhesive properties of proteolipid protein are responsible for the compaction of CNS myelin sheaths. J Neurosci 1995, 15:5502-5513.

48. Banerjee S, Sousa AD, Bhat MA: Organization and function of septate junctions: an evolutionary perspective. Cell Biochem Biophys 2006, 46:65-77.

49. FlyTrap. http://flytrap.med.yale.edu/

50. Ensembl. http://www.ensembl.org/index.html.

doi:10.1186/1471-2202-13-78

Cite this article as: Zappia et al:: A role for the membrane protein M6 in the Drosophila visual system. BMC Neuroscience 2012 13:78.

\section{Submit your next manuscript to BioMed Central and take full advantage of:}

- Convenient online submission

- Thorough peer review

- No space constraints or color figure charges

- Immediate publication on acceptance

- Inclusion in PubMed, CAS, Scopus and Google Scholar

- Research which is freely available for redistribution 